\title{
2-IRREDUCIBLE AND STRONGLY 2-IRREDUCIBLE IDEALS OF COMMUTATIVE RINGS
}

\author{
H. MOSTAFANASAB AND A. YOUSEFIAN DARANI
}

Received 08 January, 2015

\begin{abstract}
An ideal $I$ of a commutative ring $R$ is said to be irreducible if it cannot be written as the intersection of two larger ideals. A proper ideal $I$ of a ring $R$ is said to be strongly irreducible if for each ideals $J, K$ of $R, J \cap K \subseteq I$ implies that $J \subseteq I$ or $K \subseteq I$. In this paper, we introduce the concepts of 2-irreducible and strongly 2-irreducible ideals which are generalizations of irreducible and strongly irreducible ideals, respectively. We say that a proper ideal $I$ of a ring $R$ is 2-irreducible if for each ideals $J, K$ and $L$ of $R, I=J \cap K \cap L$ implies that either $I=J \cap K$ or $I=J \cap L$ or $I=K \cap L$. A proper ideal $I$ of a ring $R$ is called strongly 2-irreducible if for each ideals $J, K$ and $L$ of $R, J \cap K \cap L \subseteq I$ implies that either $J \cap K \subseteq I$ or $J \cap L \subseteq I$ or $K \cap L \subseteq I$.

2010 Mathematics Subject Classification: 13A15; 13C05; 13F05
\end{abstract}

Keywords: irreducible ideals, 2-irreducible ideals, strongly 2-irreducible ideals

\section{INTRODUCTION}

Throughout this paper all rings are commutative with a nonzero identity. Recall that an ideal $I$ of a commutative ring $R$ is irreducible if $I=J \cap K$ for ideals $J$ and $K$ of $R$ implies that either $I=J$ or $I=K$. A proper ideal $I$ of a ring $R$ is said to be strongly irreducible if for each ideals $J, K$ of $R, J \cap K \subseteq I$ implies that $J \subseteq I$ or $K \subseteq I$ (see [3], [13]). Obviously a proper ideal $I$ of a ring $R$ is strongly irreducible if and only if for each $x, y \in R, R x \cap R y \subseteq I$ implies that $x \in I$ or $y \in I$. It is easy to see that any strongly irreducible ideal is an irreducible ideal. Now, we recall some definitions which are the motivation of our work. Badawi in [4] generalized the concept of prime ideals in a different way. He defined a nonzero proper ideal $I$ of $R$ to be a 2-absorbing ideal of $R$ if whenever $a, b, c \in R$ and $a b c \in I$, then $a b \in I$ or $a c \in I$ or $b c \in I$. It is shown that a proper ideal $I$ of $R$ is a 2-absorbing ideal if and only if whenever $I_{1} I_{2} I_{3} \subseteq I$ for some ideals $I_{1}, I_{2}, I_{3}$ of $R$, then $I_{1} I_{2} \subseteq I$ or $I_{1} I_{3} \subseteq I$ or $I_{2} I_{3} \subseteq I$. In [9], Yousefian Darani and Puczyłowski studied the concept of 2-absorbing commutative semigroups. Anderson and Badawi [2] generalized the concept of 2-absorbing ideals to $n$-absorbing ideals. According to their definition, a proper ideal $I$ of $R$ is called an $n$-absorbing (resp. strongly $n$-absorbing) ideal 
if whenever $a_{1} \cdots a_{n+1} \in I$ for $a_{1}, \ldots, a_{n+1} \in R$ (resp. $I_{1} \cdots I_{n+1} \subseteq I$ for ideals $I_{1}, \cdots I_{n+1}$ of $R$ ), then there are $n$ of the $a_{i}$ 's (resp. $n$ of the $I_{i}$ 's) whose product is in $I$. Thus a strongly 1 -absorbing ideal is just a prime ideal. Clearly a strongly $n$-absorbing ideal of $R$ is also an $n$-absorbing ideal of $R$. The concept of 2-absorbing primary ideals, a generalization of primary ideals was introduced and investigated in [6]. A proper ideal $I$ of a commutative ring $R$ is called a 2-absorbing primary ideal if whenever $a, b, c \in R$ and $a b c \in I$, then either $a b \in I$ or $a c \in \sqrt{I}$ or $b c \in \sqrt{I}$. We refer the readers to [5] for a specific kind of 2-absorbing ideals and to [19], [10], [11] for the module version of the above definitions. We define an ideal $I$ of a ring $R$ to be 2-irreducible if whenever $I=J \cap K \cap L$ for ideals $I, J$ and $K$ of $R$, then either $I=J \cap K$ or $I=J \cap L$ or $I=K \cap L$. Obviously, any irreducible ideal is a 2 -irreducible ideal. Also, we say that a proper ideal $I$ of a ring $R$ is called strongly 2irreducible if for each ideals $J, K$ and $L$ of $R, J \cap K \cap L \subseteq I$ implies that $J \cap K \subseteq I$ or $J \cap L \subseteq I$ or $K \cap L \subseteq I$. Clearly, any strongly irreducible ideal is a strongly 2irreducible ideal. In [8], [7] we can find the notion of 2-irreducible preradicals and its dual, the notion of co-2-irreducible preradicals. We call a proper ideal $I$ of a ring $R$ singly strongly 2-irreducible if for each $x, y, z \in R, R x \cap R y \cap R z \subseteq I$ implies that $R x \cap R y \subseteq I$ or $R x \cap R z \subseteq I$ or $R y \cap R z \subseteq I$. It is trivial that any strongly 2-irreducible ideal is a singly strongly 2 -irreducible ideal. A ring $R$ is said to be an arithmetical ring, if for each ideals $I, J$ and $K$ of $R,(I+J) \cap K=(I \cap K)+$ $(J \cap K)$. This condition is equivalent to the condition that for each ideals $I, J$ and $K$ of $R,(I \cap J)+K=(I+K) \cap(J+K)$, see [15]. In this paper we prove that, a nonzero ideal $I$ of a principal ideal domain $R$ is 2-irreducible if and only if $I$ is strongly 2-irreducible if and only if $I$ is 2 -absorbing primary. It is shown that a proper ideal $I$ of a ring $R$ is strongly 2-irreducible if and only if for each $x, y, z \in R$, $(R x+R y) \cap(R x+R z) \cap(R y+R z) \subseteq I$ implies that $(R x+R y) \cap(R x+R z) \subseteq I$ or $(R x+R y) \cap(R y+R z) \subseteq I$ or $(R x+R z) \cap(R y+R z) \subseteq I$. A proper ideal $I$ of a von Neumann regular ring $R$ is 2-irreducible if and only if $I$ is 2-absorbing if and only if for every idempotent elements $e_{1}, e_{2}, e_{3}$ of $R, e_{1} e_{2} e_{3} \in I$ implies that either $e_{1} e_{2} \in I$ or $e_{1} e_{3} \in I$ or $e_{2} e_{3} \in I$. If $I$ is a 2-irreducible ideal of a Noetherian ring $R$, then $I$ is a 2-absorbing primary ideal of $R$. Let $R=R_{1} \times R_{2}$, where $R_{1}$ and $R_{2}$ are commutative rings with $1 \neq 0$. It is shown that a proper ideal $J$ of $R$ is a strongly 2-irreducible ideal of $R$ if and only if either $J=I_{1} \times R_{2}$ for some strongly 2-irreducible ideal $I_{1}$ of $R_{1}$ or $J=R_{1} \times I_{2}$ for some strongly 2-irreducible ideal $I_{2}$ of $R_{2}$ or $J=I_{1} \times I_{2}$ for some strongly irreducible ideal $I_{1}$ of $R_{1}$ and some strongly irreducible ideal $I_{2}$ of $R_{2}$. A proper ideal $I$ of a unique factorization domain $R$ is singly strongly 2-irreducible if and only if $p_{1}^{n_{1}} p_{2}^{n_{2}} \cdots p_{k}^{n_{k}} \in I$, where $p_{i}$ 's are distinct prime elements of $R$ and $n_{i}$ 's are natural numbers, implies that $p_{r}^{n_{r}} p_{s}^{n_{s}} \in I$, for some $1 \leq r, s \leq k$. 


\section{BASIC PROPERTIES OF 2-IRREDUCIBLE AND STRONGLY 2-IRREDUCIBLE IDEALS}

It is important to notice that when $R$ is a domain, then $R$ is an arithmetical ring if and only if $R$ is a Prüfer domain. In particular, every Dedekind domain is an arithmetical domain.

Theorem 1. Let $R$ be a Dedekind domain and $I$ be a nonzero proper ideal of $R$. The following conditions are equivalent:

(1) I is a strongly irreducible ideal;

(2) I is an irreducible ideal;

(3) I is a primary ideal;

(4) $I=R p^{n}$ for some prime (irreducible) element $p$ of $R$ and some natural number $n$.

Proof. See [13, Lemma 2.2(3)] and [18, p. 130, Exercise 36].

We recall from [1] that an integral domain $R$ is called a $G C D$-domain if any two nonzero elements of $R$ have a greatest common divisor (GCD), equivalently, any two nonzero elements of $R$ have a least common multiple ( $L C M)$. Unique factorization domains (UFD's) are well-known examples of $G C D$-domains. Let $R$ be a $G C D$-domain. The least common multiple of elements $x, y$ of $R$ is denoted by $[x, y]$. Notice that for every elements $x, y \in R, R x \cap R y=R[x, y]$. Moreover, for every elements $x, y, z$ of $R$, we have $[[x, y], z]=[x,[y, z]]$. So we denote $[[x, y], z]$ simply by $[x, y, z]$.

Recall that every principal ideal domain $(P I D)$ is a Dedekind domain.

Theorem 2. Let $R$ be a PID and I be a nonzero proper ideal of $R$. The following conditions are equivalent:

(1) I is a 2-irreducible ideal;

(2) I is a 2-absorbing primary ideal;

(3) Either $I=R p^{k}$ for some prime (irreducible) element $p$ of $R$ and some natural number $n$, or $I=R\left(p_{1}^{n} p_{2}^{m}\right)$ for some distinct prime (irreducible) elements $p_{1}, p_{2}$ of $R$ and some natural numbers $n, m$.

Proof. (2) $\Leftrightarrow$ (3) See [6, Corollary 2.12].

(1) $\Rightarrow$ (3) Assume that $I=R a$ where $0 \neq a \in R$. Let $a=p_{1}^{n_{1}} p_{2}^{n_{2}} \cdots p_{k}^{n_{k}}$ be a prime decomposition for $a$. We show that either $k=1$ or $k=2$. Suppose that $k>2$. By [14, p. 141, Exercise 5], we have that $I=R p_{1}^{n_{1}} \cap R p_{2}^{n_{2}} \cap \cdots \cap R p_{k}^{n_{k}}$. Now, since $I$ is 2-irreducible, there exist $1 \leq i, j \leq k$ such that $I=R p_{i}^{n_{i}} \cap R p_{j}^{n_{j}}$, say $i=1, j=2$. Therefore we have $I=R p_{1}^{n_{1}} \cap R p_{2}^{n_{2}} \subseteq R p_{3}^{n_{3}}$, which is a contradiction.

(3) $\Rightarrow$ (1) If $I=R p^{k}$ for some prime element $p$ of $R$ and some natural number $n$, then $I$ is irreducible, by Theorem 1 , and so $I$ is 2-irreducible. Therefore, assume 
that $I=R\left(p_{1}^{n} p_{2}^{m}\right)$ for some distinct prime elements $p_{1}, p_{2}$ of $R$ and some natural numbers $n, m$. Let $I=R a \cap R b \cap R c$ for some elements $a, b$ and $c$ of $R$. Then $a, b$ and $c$ divide $p_{1}^{n} p_{2}^{m}$, and so $a=p_{1}^{\alpha_{1}} p_{2}^{\alpha_{2}}, b=p_{1}^{\beta_{1}} p_{2}^{\beta_{2}}$ and $c=p_{1}^{\gamma_{1}} p_{2}^{\gamma_{2}}$ where $\alpha_{i}, \beta_{i}, \gamma_{i}$ are some nonnegative integers. On the other hand $I=R a \cap R b \cap R c=$ $R[a, b, c]=R\left(p_{1}^{\delta} p_{2}^{\varepsilon}\right)$ in which $\delta=\max \left\{\alpha_{1}, \beta_{1}, \gamma_{1}\right\}$ and $\varepsilon=\max \left\{\alpha_{2}, \beta_{2}, \gamma_{2}\right\}$. We can assume without loss of generality that $\delta=\alpha_{1}$ and $\varepsilon=\beta_{2}$. So $I=R\left(p_{1}^{\alpha_{1}} p_{2}^{\beta_{2}}\right)=$ $R a \cap R b$. Consequently, $I$ is 2-irreducible.

A commutative ring $R$ is called a von Neumann regular ring (or an absolutely flat ring) if for any $a \in R$ there exists an $x \in R$ with $a^{2} x=a$, equivalently, $I=I^{2}$ for every ideal $I$ of $R$.

Remark 1. Notice that a commutative ring $R$ is a von Neumann regular ring if and only if $I J=I \cap J$ for any ideals $I, J$ of $R$, by [16, Lemma 1.2]. Therefore over a commutative von Neumann regular ring the two concepts of strongly 2 -irreducible ideals and of 2-absorbing ideals are coincide.

Theorem 3. Let I be a proper ideal of a ring $R$. Then the following conditions are equivalent:

(1) I is strongly 2-irreducible;

(2) For every elements $x, y, z$ of $R,(R x+R y) \cap(R x+R z) \cap(R y+R z) \subseteq I$ implies that $(R x+R y) \cap(R x+R z) \subseteq I$ or $(R x+R y) \cap(R y+R z) \subseteq I$ or $(R x+R z) \cap(R y+R z) \subseteq I$.

Proof. (1) $\Rightarrow(2)$ There is nothing to prove.

(2) $\Rightarrow$ (1) Suppose that $J, K$ and $L$ are ideals of $R$ such that neither $J \cap K \subseteq I$ nor $J \cap L \subseteq I$ nor $K \cap L \subseteq I$. Then there exist elements $x, y$ and $z$ of $R$ such that $x \in(J \cap K) \backslash I$ and $y \in(J \cap L) \backslash I$ and $z \in(K \cap L) \backslash I$. On the other hand $(R x+R y) \cap(R x+R z) \cap(R y+R z) \subseteq(R x+R y) \subseteq J,(R x+R y) \cap(R x+R z) \cap$ $(R y+R z) \subseteq(R x+R z) \subseteq K$ and $(R x+R y) \cap(R x+R z) \cap(R y+R z) \subseteq(R y+$ $R z) \subseteq L$. Hence $(R x+R y) \cap(R x+R z) \cap(R y+R z) \subseteq I$, and so by hypothesis either $(R x+R y) \cap(R x+R z) \subseteq I$ or $(R x+R y) \cap(R y+R z) \subseteq I$ or $(R x+R z) \cap$ $(R y+R z) \subseteq I$. Therefore, either $x \in I$ or $y \in I$ or $z \in I$, which any of these cases has a contradiction. Consequently $I$ is strongly 2 -irreducible.

A ring $R$ is called a Bézout ring if every finitely generated ideal of $R$ is principal. As an immediate consequence of Theorem 3 we have the next result:

Corollary 1. Let I be a proper ideal of a Bézout ring $R$. Then the following conditions are equivalent:

(1) I is strongly 2-irreducible;

(2) I is singly strongly 2-irreducible;

Now we can state the following open problem. 
Problem 1. Let I be a singly strongly 2-irreducible ideal of a ring $R$. Is I a strongly 2-irreducible ideal of $R$ ?

Proposition 1. Let $R$ be a ring. If I is a strongly 2-irreducible ideal of $R$, then I is a 2-irreducible ideal of $R$.

Proof. Suppose that $I$ is a strongly 2-irreducible ideal of $R$. Let $J, K$ and $L$ be ideals of $R$ such that $I=J \cap K \cap L$. Since $J \cap K \cap L \subseteq I$, then either $J \cap K \subseteq I$ or $J \cap L \subseteq I$ or $K \cap L \subseteq I$. On the other hand $I \subseteq J \cap K$ and $I \subseteq J \cap L$ and $I \subseteq K \cap L$. Consequently, either $I=J \cap K$ or $I=J \cap L$ or $I=K \cap L$. Therefore $I$ is 2-irreducible.

Remark 2. It is easy to check that the zero ideal $I=\{0\}$ of a ring $R$ is 2-irreducible if and only if $I$ is strongly 2 -irreducible.

Proposition 2. Let I be a proper ideal of an arithmetical ring $R$. The following conditions are equivalent:

(1) I is a 2-irreducible ideal of $R$;

(2) $I$ is a strongly 2-irreducible ideal of $R$;

(3) For every ideals $I_{1}, I_{2}$ and $I_{3}$ of $R$ with $I \subseteq I_{1}, I_{1} \cap I_{2} \cap I_{3} \subseteq I$ implies that $I_{1} \cap I_{2} \subseteq I$ or $I_{1} \cap I_{3} \subseteq I$ or $I_{2} \cap I_{3} \subseteq I$.

Proof. (1) $\Rightarrow$ (2) Assume that $J, K$ and $L$ are ideals of $R$ such that $J \cap K \cap L \subseteq$ $I$. Therefore $I=I+(J \cap K \cap L)=(I+J) \cap(I+K) \cap(I+L)$, since $R$ is an arithmetical ring. So either $I=(I+J) \cap(I+K)$ or $I=(I+J) \cap(I+L)$ or $I=(I+K) \cap(I+L)$, and thus either $J \cap K \subseteq I$ or $J \cap L \subseteq I$ or $K \cap L \subseteq I$. Hence $I$ is a strongly 2 -irreducible ideal.

$(2) \Rightarrow(3)$ is clear.

(3) $\Rightarrow$ (2) Let $J, K$ and $L$ be ideals of $R$ such that $J \cap K \cap L \subseteq I$. Set $I_{1}:=J+I$, $I_{2}:=K$ and $I_{3}:=L$. Since $R$ is an arithmetical ring, then $I_{1} \cap I_{2} \cap I_{3}=(J+I) \cap$ $K \cap L=(J \cap K \cap L)+(I \cap K \cap L) \subseteq I$. Hence either $I_{1} \cap I_{2} \subseteq I$ or $I_{1} \cap I_{3} \subseteq I$ or $I_{2} \cap I_{3} \subseteq I$ which imply that either $J \cap K \subseteq I$ or $J \cap L \subseteq I$ or $K \cap L \subseteq I$, respectively. Consequently, $I$ is a strongly 2-irreducible ideal of $R$.

(2) $\Rightarrow(1)$ By Proposition 1.

As an immediate consequence of Theorem 2 and Proposition 2 we have the next result.

Corollary 2. Let $R$ be a PID and I be a nonzero proper ideal of $R$. The following conditions are equivalent:

(1) I is a strongly 2-irreducible ideal;

(2) I is a 2-irreducible ideal;

(3) I is a 2-absorbing primary ideal;

(4) Either $I=R p^{k}$ for some prime (irreducible) element $p$ of $R$ and some natural number $n$, or $I=R\left(p_{1}^{n} p_{2}^{m}\right)$ for some distinct prime (irreducible) elements $p_{1}, p_{2}$ of $R$ and some natural numbers $n, m$. 
The following example shows that the concepts of strongly irreducible (irreducible) ideals and of strongly 2-irreducible (2-irreducible) ideals are different in general.

Example 1. Consider the ideal $6 \mathbb{Z}$ of the ring $\mathbb{Z}$. By Corollary $2,6 \mathbb{Z}=(2.3) \mathbb{Z}$ is a strongly 2 -irreducible (a 2 -irreducible) ideal of $\mathbb{Z}$. But, Theorem 1 says that $6 \mathbb{Z}$ is not a strongly irreducible (an irreducible) ideal of $\mathbb{Z}$.

It is well known that every von Neumann regular ring is a Bézout ring. By [15, p. $119]$, every Bézout ring is an arithmetical ring.

Corollary 3. Let I be a proper ideal of a von Neumann regular ring $R$. The following conditions are equivalent:

(1) I is a 2-absorbing ideal of $R$;

(2) $I$ is a 2-irreducible ideal of $R$;

(3) I is a strongly 2-irreducible ideal of $R$;

(4) $I$ is a singly strongly 2-irreducible of $R$;

(5) For every idempotent elements $e_{1}, e_{2}, e_{3}$ of $R, e_{1} e_{2} e_{3} \in I$ implies that either $e_{1} e_{2} \in I$ or $e_{1} e_{3} \in I$ or $e_{2} e_{3} \in I$.

Proof. (1) $\Leftrightarrow$ (3) By Remark 1.

$(2) \Leftrightarrow(3)$ By Proposition 2.

(3) $\Leftrightarrow$ (4) By Corollary 1.

$(1) \Rightarrow(5)$ is evident.

$(5) \Rightarrow(3)$ The proof follows from Theorem 3 and the fact that any finitely generated ideal of a von Neumann regular ring $R$ is generated by an idempotent element.

Proposition 3. Let $I_{1}, I_{2}$ be strongly irreducible ideals of a ring $R$. Then $I_{1} \cap I_{2}$ is a strongly 2-irreducible ideal of $R$.

Proof. Strightforward.

Theorem 4. Let $R$ be a Noetherian ring. If $I$ is a 2-irreducible ideal of $R$, then either I is irreducible or I is the intersection of exactly two irreducible ideals. The converse is true when $R$ is also arithmetical.

Proof. Assume that $I$ is 2-irreducible. By [20, Proposition 4.33], $I$ can be written as a finite irredundant irreducible decomposition $I=I_{1} \cap I_{2} \cap \cdots \cap I_{k}$. We show that either $k=1$ or $k=2$. If $k>3$, then since $I$ is 2-irreducible, $I=I_{i} \cap I_{j}$ for some $1 \leq i, j \leq k$, say $i=1$ and $j=2$. Therefore $I_{1} \cap I_{2} \subseteq I_{3}$, which is a contradiction. For the second atatement, let $R$ be arithmetical, and $I$ be the intersection of two irreducible ideals. Since $R$ is arithmetical, every irreducible ideal is strongly irreducible, [13, Lemma 2.2(3)]. Now, apply Proposition 3 to see that $I$ is strongly 2-irreducible, and so $I$ is 2-irreducible.

Corollary 4. Let $R$ be a Noetherian ring and $I$ be a proper ideal of $R$. If $I$ is 2-irreducible, then $I$ is a 2-absorbing primary ideal of $R$. 
Proof. Assume that $I$ is 2 -irreducible. By the fact that every irreducible ideal of a Noetherian ring is primary and regarding Theorem 4, we have either $I$ is a primary ideal or is the intersection of two primary ideals. It is clear that every primary ideal is 2-absorbing primary, also the intersection of two primary ideals is a 2-absorbing primary ideal, by [6, Theorem 2.4].

Proposition 4. Let $R$ be a ring, and let $P_{1}, P_{2}$ and $P_{3}$ be pairwise comaximal prime ideals of $R$. Then $P_{1} P_{2} P_{3}$ is not a 2-irreducible ideal.

Proof. The proof is easy.

Corollary 5. If $R$ is a ring such that every proper ideal of $R$ is 2-irreducible, then $R$ has at most two maximal ideals.

Theorem 5. Let I be a radical ideal of a ring $R$, i.e., $I=\sqrt{I}$. The following conditions are equivalent:

(1) I is strongly 2-irreducible;

(2) I is 2-absorbing;

(3) I is 2-absorbing primary;

(4) I is either a prime ideal of $R$ or is an intersection of exactly two prime ideals of $R$.

Proof. (1) $\Rightarrow$ (2) Assume that $I$ is strongly 2-irreducible. Let $J, K$ and $L$ be ideals of $R$ such that $J K L \subseteq I$. Then $J \cap K \cap L \subseteq \sqrt{J \cap K \cap L} \subseteq \sqrt{I}=I$. So, either $J \cap K \subseteq I$ or $J \cap L \subseteq I$ or $K \cap L \subseteq I$. Hence either $J K \subseteq I$ or $J L \subseteq I$ or $K L \subseteq I$. Consequently $I$ is 2 -absorbing.

$(2) \Leftrightarrow(3)$ is obvious.

$(2) \Rightarrow(4)$ If $I$ is a 2 -absorbing ideal, then either $\sqrt{I}$ is a prime ideal or is an intersection of exactly two prime ideals, [4, Theorem 2.4]. Now, we prove the claim by assumption that $I=\sqrt{I}$.

(4) $\Rightarrow$ (1) By Proposition 3.

Theorem 6. Let $f: R \rightarrow S$ be a surjective homomorphism of commutative rings, and let $I$ be an ideal of $R$ containing $\operatorname{Ker}(f)$. Then,

(1) If I is a strongly 2-irreducible ideal of $R$, then $I^{e}$ is a strongly 2-irreducible ideal of $S$.

(2) $I$ is a 2-irreducible ideal of $R$ if and only if $I^{e}$ is a 2-irreducible ideal of $S$.

Proof. Since $f$ is surjective, $J^{c e}=J$ for every ideal $J$ of $S$. Moreover, $(K \cap$ $L)^{e}=K^{e} \cap L^{e}$ and $K^{e c}=K$ for every ideals $K, L$ of $R$ which contain $\operatorname{Ker}(\mathrm{f})$.

(1) Suppose that $I$ is a strongly 2-irreducible ideal of $R$. If $I^{e}=S$, then $I=I^{e c}=R$, which is a contradiction. Let $J_{1}, J_{2}$ and $J_{3}$ be ideals of $S$ such that $J_{1} \cap J_{2} \cap J_{3} \subseteq I^{e}$. Therefore $J_{1}^{c} \cap J_{2}^{c} \cap J_{3}^{c} \subseteq I^{e c}=I$. So, either $J_{1}^{c} \cap J_{2}^{c} \subseteq I$ or $J_{1}^{c} \cap J_{3}^{c} \subseteq I$ or $J_{2}^{c} \cap J_{3}^{c} \subseteq I$. Without loss of generality, we may assume that $J_{1}^{c} \cap J_{2}^{c} \subseteq I$. So, $J_{1} \cap J_{2}=\left(J_{1} \cap J_{2}\right)^{c e} \subseteq I^{e}$. Hence $I^{e}$ is strongly 2-irreducible. 
(2) The necessity is similar to part (1). Conversely, let $I^{e}$ be a strongly 2 -irreducible ideal of $S$, and let $I_{1}, I_{2}$ and $I_{3}$ be ideals of $R$ such that $I=I_{1} \cap I_{2} \cap I_{3}$. Then $I^{e}=I_{1}^{e} \cap I_{2}^{e} \cap I_{3}^{e}$. Hence, either $I^{e}=I_{1}^{e} \cap I_{2}^{e}$ or $I^{e}=I_{1}^{e} \cap I_{3}^{e}$ or $I^{e}=I_{2}^{e} \cap I_{3}^{e}$. We may assume that $I^{e}=I_{1}^{e} \cap I_{2}^{e}$. Therefore, $I=I^{e c}=I_{1}^{e c} \cap I_{2}^{e c}=I_{1} \cap I_{2}$. Consequently, $I$ is strongly 2-irreducible.

Corollary 6. Let $f: R \rightarrow S$ be a surjective homomorphism of commutative rings. There is a one-to-one correspondence between the 2-irreducible ideals of $R$ which contain $\operatorname{Ker}(f)$ and 2-irreducible ideals of $S$.

Recall that a ring $R$ is called a Laskerian ring if every proper ideal of $R$ has a primary decomposition. Noetherian rings are some examples of Laskerian rings.

Let $S$ be a multiplicatively closed subset of a ring $R$. In the next theorem, consider the natural homomorphism $f: R \rightarrow S^{-1} R$ defined by $f(x)=x / 1$.

Theorem 7. Let I be a proper ideal of a ring $R$ and $S$ be a multiplicatively closed set in $R$.

(1) If $I$ is a strongly 2-irreducible ideal of $S^{-1} R$, then $I^{c}$ is a strongly 2irreducible ideal of $R$.

(2) If $I$ is a primary strongly 2-irreducible ideal of $R$ such that $I \cap S=\varnothing$, then $I^{e}$ is a strongly 2-irreducible ideal of $S^{-1} R$.

(3) If $I$ is a primary ideal of $R$ such that $I^{e}$ is a strongly 2-irreducible ideal of $S^{-1} R$, then $I$ is a strongly 2-irreducible ideal of $R$.

(4) If $R^{\prime}$ is a faithfully flat extension ring of $R$ and if $I R^{\prime}$ is a strongly 2-irreducible ideal of $R^{\prime}$, then $I$ is a strongly 2-irreducible ideal of $R$.

(5) If $I$ is strongly 2-irreducible and $H$ is an ideal of $R$ such that $H \subseteq I$, then $I / H$ is a strongly 2-irreducible ideal of $R / H$.

(6) If $R$ is a Laskerian ring, then every strongly 2-irreducible ideal is either a primary ideal or is the intersection of two primary ideals.

Proof. (1) Assume that $I$ is a strongly 2-irreducible ideal of $S^{-1} R$. Let $J, K$ and $L$ be ideals of $R$ such that $J \cap K \cap L \subseteq I^{c}$. Then $J^{e} \cap K^{e} \cap L^{e} \subseteq I^{c e}=I$. Hence either $J^{e} \cap K^{e} \subseteq I$ or $J^{e} \cap L^{e} \subseteq I$ or $K^{e} \cap L^{e} \subseteq I$ since $I$ is strongly 2-irreducible. Therefore either $J \cap K \subseteq I^{c}$ or $J \cap L \subseteq I^{c}$ or $K \cap L \subseteq I^{c}$. Consequently $I^{c}$ is a strongly 2-irreducible ideal of $R$.

(2) Suppose that $I$ is a primary strongly 2-irreducible ideal such that $I \cap S=\varnothing$. Let $J, K$ and $L$ be ideals of $S^{-1} R$ such that $J \cap K \cap L \subseteq I^{e}$. Since $I$ is a primary ideal, then $J^{c} \cap K^{c} \cap L^{c} \subseteq I^{e c}=I$. Thus $J^{c} \cap K^{c} \subseteq I$ or $J^{c} \cap L^{c} \subseteq I$ or $K^{c} \cap L^{c} \subseteq I$. Hence $J \cap K \subseteq I^{e}$ or $J \cap L \subseteq I^{e}$ or $K \cap L \subseteq I^{e}$.

(3) Let $I$ be a primary ideal of $R$, and let $I^{e}$ be a strongly 2-irreducible ideal of $S^{-1} R$. By part (1), $I^{e c}$ is strongly 2-irreducible. Since $I$ is primary, we have $I^{e c}=I$, and thus we are done.

(4) Let $J, K$ and $L$ be ideals of $R$ such that $J \cap K \cap L \subseteq I$. Thus $J R^{\prime} \cap K R^{\prime} \cap L R^{\prime}=$ $(J \cap K \cap L) R^{\prime} \subseteq I R^{\prime}$, by [12, Lemma 9.9]. Since $I R^{\prime}$ is strongly 2-irreducible, then 
either $J R^{\prime} \cap K R^{\prime} \subseteq I R^{\prime}$ or $J R^{\prime} \cap L R^{\prime} \subseteq I R^{\prime}$ or $K R^{\prime} \cap L R^{\prime} \subseteq I R^{\prime}$. Without loss of generality, assume that $J R^{\prime} \cap K R^{\prime} \subseteq I R^{\prime}$. So, $\left(J R^{\prime} \cap R\right) \cap\left(K R^{\prime} \cap R\right) \subseteq I R^{\prime} \cap R$. Hence $J \cap K \subseteq I$, by [17, Theorem 4.74]. Consequently $I$ is strongly 2-irreducible. (5) Let $J, K$ and $L$ be ideals of $R$ containing $H$ such that $(J / H) \cap(K / H) \cap$ $(L / H) \subseteq I / H$. Hence $J \cap K \cap L \subseteq I$. Therefore, either $J \cap K \subseteq I$ or $J \cap L \subseteq$ $I$ or $K \cap L \subseteq I$. Thus, $(J / H) \cap(K / H) \subseteq I / H$ or $(J / H) \cap(L / H) \subseteq I / H$ or $(K / H) \cap(L / H) \subseteq I / H$. Consequently, $I / H$ is strongly 2 -irreducible.

(6) Let $I$ be a strongly 2-irreducible ideal and $\cap_{i=1}^{n} Q_{i}$ be a primary decomposition of $I$. Since $\cap_{i=1}^{n} Q_{i} \subseteq I$, then there are $1 \leq r, s \leq n$ such that $Q_{r} \cap Q_{s} \subseteq I=$ $\cap_{i=1}^{n} Q_{i} \subseteq Q_{r} \cap Q_{s}$.

Let $S$ be a multiplicatively closed subset of a ring $R$. Set

$$
C:=\left\{I^{c} \mid I \text { is an ideal of } R_{S}\right\} .
$$

Corollary 7. Let $R$ be a ring and $S$ be a multiplicatively closed subset of $R$. Then there is a one-to-one correspondence between the strongly 2-irreducible ideals of $R_{S}$ and strongly 2-irreducible ideals of $R$ contained in $C$ which do not meet $S$.

Proof. If $I$ is a strongly 2 -irreducible ideal of $R_{S}$, then evidently $I^{c} \neq R, I^{c} \in C$ and by Theorem 7(1), $I^{c}$ is a strongly 2 -irreducible ideal of $R$. Conversely, let $I$ be a strongly 2-irreducible ideal of $R, I \cap S=\varnothing$ and $I \in C$. Since $I \cap S=\varnothing, I^{e} \neq R_{S}$. Let $J \cap K \cap L \subseteq I^{e}$ where $J, K$ and $L$ are ideals of $R_{S}$. Then $J^{c} \cap K^{c} \cap L^{c}=(J \cap$ $K \cap L)^{c} \subseteq I^{e c}$. Now since $I \in C$, then $I^{e c}=I$. So $J^{c} \cap K^{c} \cap L^{c} \subseteq I$. Hence, either $J^{c} \cap K^{c} \subseteq I$ or $J^{c} \cap L^{c} \subseteq I$ or $K^{c} \cap L^{c} \subseteq I$. Then, either $J \cap K=(J \cap K)^{c e} \subseteq I^{e}$ or $J \cap L=(J \cap L)^{c e} \subseteq I^{e}$ or $K \cap L=(K \cap L)^{c e} \subseteq I^{e}$. Consequently, $I^{e}$ is a strongly 2-irreducible ideal of $R_{S}$.

Let $n$ be a natural number. We say that $I$ is an $n$-primary ideal of a ring $R$ if $I$ is the intersection of $n$ primary ideals of $R$.

Proposition 5. Let $R$ be a ring. Then the following conditions are equivalent:

(1) Every n-primary ideal of $R$ is a strongly 2-irreducible ideal;

(2) For any prime ideal $P$ of $R$, every n-primary ideal of $R_{P}$ is a strongly 2irreducible ideal;

(3) For any maximal ideal $m$ of $R$, every $n$-primary ideal of $R_{m}$ is a strongly 2-irreducible ideal.

Proof. (1) $\Rightarrow(2)$ Let $I$ be an $n$-primary ideal of $R_{P}$. We know that $I^{c}$ is an $n$ primary ideal of $R, I^{c} \cap(R \backslash P)=\varnothing, I^{c} \in C$ and, by the assumption, $I^{c}$ is a strongly 2-irreducible ideal of $R$. Now, by Corollary $7, I=\left(I^{c}\right)_{P}$ is a strongly 2-irreducible ideal of $R_{P}$.

$(2) \Rightarrow(3)$ is clear.

(3) $\Rightarrow(1)$ Let $I$ be an $n$-primary ideal of $R$ and let $m$ be a maximal ideal of $R$ containing $I$. Then, $I_{m}$ is an $n$-primary ideal of $R_{m}$ and so, by our assumption, $I_{m}$ is 
a strongly 2-irreducible ideal of $R_{m}$. Now by Theorem 10(1), $\left(I_{m}\right)^{c}$ is a strongly 2-irreducible ideal of $R$, and since $I$ is an $n$-primary ideal of $R,\left(I_{m}\right)^{c}=I$, that is, $I$ is a strongly 2 -irreducible ideal of $R$.

Theorem 8. Let $R=R_{1} \times R_{2}$, where $R_{1}$ and $R_{2}$ are rings with $1 \neq 0$. Let $J$ be a proper ideal of $R$. Then the following conditions are equivalent:

(1) $J$ is a strongly 2-irreducible ideal of $R$;

(2) Either $J=I_{1} \times R_{2}$ for some strongly 2-irreducible ideal $I_{1}$ of $R_{1}$ or $J=$ $R_{1} \times I_{2}$ for some strongly 2-irreducible ideal $I_{2}$ of $R_{2}$ or $J=I_{1} \times I_{2}$ for some strongly irreducible ideal $I_{1}$ of $R_{1}$ and some strongly irreducible ideal $I_{2}$ of $R_{2}$.

Proof. (1) $\Rightarrow(2)$ Assume that $J$ is a strongly 2-irreducible ideal of $R$. Then $J=$ $I_{1} \times I_{2}$ for some ideal $I_{1}$ of $R_{1}$ and some ideal $I_{2}$ of $R_{2}$. Suppose that $I_{2}=R_{2}$. Since $J$ is a proper ideal of $R, I_{1} \neq R_{1}$. Let $R^{\prime}=\frac{R}{\{0\} \times R_{2}}$. Then $J^{\prime}=\frac{J}{\{0\} \times R_{2}}$ is a strongly 2-irreducible ideal of $R^{\prime}$ by Theorem 7(5). Since $R^{\prime}$ is ring-isomorphic to $R_{1}$ and $I_{1} \simeq J^{\prime}, I_{1}$ is a strongly 2 -irreducible ideal of $R_{1}$. Suppose that $I_{1}=R_{1}$. Since $J$ is a proper ideal of $R, I_{2} \neq R_{2}$. By a similar argument as in the previous case, $I_{2}$ is a strongly 2-irreducible ideal of $R_{2}$. Hence assume that $I_{1} \neq R_{1}$ and $I_{2} \neq R_{2}$. Suppose that $I_{1}$ is not a strongly irreducible ideal of $R_{1}$. Then there are $x, y \in R_{1}$ such that $R_{1} x \cap R_{1} y \subseteq I_{1}$ but neither $x \in I_{1}$ nor $y \in I_{1}$. Notice that $\left(R_{1} x \times R_{2}\right) \cap$ $\left(R_{1} \times\{0\}\right) \cap\left(R_{1} y \times R_{2}\right)=\left(R_{1} x \cap R_{1} y\right) \times\{0\} \subseteq J$, but neither $\left(R_{1} x \times R_{2}\right) \cap\left(R_{1} \times\right.$ $\{0\})=R_{1} x \times\{0\} \subseteq J$ nor $\left(R_{1} x \times R_{2}\right) \cap\left(R_{1} y \times R_{2}\right)=\left(R_{1} x \cap R_{1} y\right) \times R_{2} \subseteq J$ nor $\left(R_{1} \times\{0\}\right) \cap\left(R_{1} y \times R_{2}\right)=R_{1} y \times\{0\} \subseteq J$, which is a contradiction. Thus $I_{1}$ is a strongly irreducible ideal of $R_{1}$. Suppose that $I_{2}$ is not a strongly irreducible ideal of $R_{2}$. Then there are $z, w \in R_{2}$ such that $R_{2} z \cap R_{2} w \subseteq I_{2}$ but neither $z \in I_{2}$ nor $w \in$ $I_{2}$. Notice that $\left(R_{1} \times R_{2} z\right) \cap\left(\{0\} \times R_{2}\right) \cap\left(R_{1} \times R_{2} w\right)=\{0\} \times\left(R_{2} z \cap R_{2} w\right) \subseteq J$, but neither $\left(R_{1} \times R_{2} z\right) \cap\left(\{0\} \times R_{2}\right)=\{0\} \times R_{2} z \subseteq J$, nor $\left(R_{1} \times R_{2} z\right) \cap\left(R_{1} \times R_{2} w\right)=$ $R_{1} \times\left(R_{2} z \cap R_{2} w\right) \subseteq J$ nor $\left(\{0\} \times R_{2}\right) \cap\left(R_{1} \times R_{2} w\right)=\{0\} \times R_{2} w \subseteq J$, which is a contradiction. Thus $I_{2}$ is a strongly irreducible ideal of $R_{2}$.

(2) $\Rightarrow$ (1) If $J=I_{1} \times R_{2}$ for some strongly 2-irreducible ideal $I_{1}$ of $R_{1}$ or $J=R_{1} \times I_{2}$ for some strongly 2 -irreducible ideal $I_{2}$ of $R_{2}$, then it is clear that $J$ is a strongly 2irreducible ideal of $R$. Hence assume that $J=I_{1} \times I_{2}$ for some strongly irreducible ideal $I_{1}$ of $R_{1}$ and some strongly irreducible ideal $I_{2}$ of $R_{2}$. Then $I_{1}^{\prime}=I_{1} \times R_{2}$ and $I_{2}^{\prime}=R_{1} \times I_{2}$ are strongly irreducible ideals of $R$. Hence $I_{1}^{\prime} \cap I_{2}^{\prime}=I_{1} \times I_{2}=J$ is a strongly 2-irreducible ideal of $R$ by Proposition 3 .

Theorem 9. Let $R=R_{1} \times R_{2} \times \cdots \times R_{n}$, where $2 \leq n<\infty$, and $R_{1}, R_{2}, \ldots, R_{n}$ are rings with $1 \neq 0$. Let $J$ be a proper ideal of $R$. Then the following conditions are equivalent:

(1) $J$ is a strongly 2-irreducible ideal of $R$.

(2) Either $J=\times_{t=1}^{n} I_{t}$ such that for some $k \in\{1,2, \ldots, n\}, I_{k}$ is a strongly 2irreducible ideal of $R_{k}$, and $I_{t}=R_{t}$ for every $t \in\{1,2, \ldots, n\} \backslash\{k\}$ or $J=$ 
$\times_{t=1}^{n} I_{t}$ such that for some $k, m \in\{1,2, \ldots, n\}, I_{k}$ is a strongly irreducible ideal of $R_{k}, I_{m}$ is a strongly irreducible ideal of $R_{m}$, and $I_{t}=R_{t}$ for every $t \in\{1,2, \ldots, n\} \backslash\{k, m\}$.

Proof. We use induction on $n$. Assume that $n=2$. Then the result is valid by Theorem 8. Thus let $3 \leq n<\infty$ and assume that the result is valid when $K=R_{1} \times$ $\cdots \times R_{n-1}$. We prove the result when $R=K \times R_{n}$. By Theorem $8, J$ is a strongly 2irreducible ideal of $R$ if and only if either $J=L \times R_{n}$ for some strongly 2-irreducible ideal $L$ of $K$ or $J=K \times L_{n}$ for some strongly 2-irreducible ideal $L_{n}$ of $R_{n}$ or $J=L \times L_{n}$ for some strongly irreducible ideal $L$ of $K$ and some strongly irreducible ideal $L_{n}$ of $R_{n}$. Observe that a proper ideal $Q$ of $K$ is a strongly irreducible ideal of $K$ if and only if $Q=\times_{t=1}^{n-1} I_{t}$ such that for some $k \in\{1,2, \ldots, n-1\}, I_{k}$ is a strongly irreducible ideal of $R_{k}$, and $I_{t}=R_{t}$ for every $t \in\{1,2, \ldots, n-1\} \backslash\{k\}$. Thus the claim is now verified.

Lemma 1. Let $R$ be a $G C D$-domain and I be a proper ideal of $R$. The following conditions are equivalent:

(1) I is a singly strongly 2-irreducible ideal;

(2) For every elements $x, y, z \in R,[x, y, z] \in I$ implies that $[x, y] \in I$ or $[x, z] \in$ I or $[y, z] \in I$.

Proof. Since for every elements $x, y$ of $R$ we have $R x \cap R y=R[x, y]$, there is nothing to prove.

Now we study singly strongly 2 -irreducible ideals of a $U F D$.

Theorem 10. Let $R$ be a UFD, and let I be a proper ideal of $R$. Then the following conditions hold:

(1) $I$ is singly strongly 2-irreducible if and only if for each elements $x, y, z$ of $R$, $[x, y, z] \in I$ implies that either $[x, y] \in I$ or $[x, z] \in I$ or $[y, z] \in I$.

(2) I is singly strongly 2-irreducible if and only if $p_{1}^{n_{1}} p_{2}^{n_{2}} \cdots p_{k}^{n_{k}} \in I$, where $p_{i}$ 's are distinct prime elements of $R$ and $n_{i}$ 's are natural numbers, implies that $p_{r}^{n_{r}} p_{s}^{n_{s}} \in I$, for some $1 \leq r, s \leq k$.

(3) If $I$ is a nonzero principal ideal, then I is singly strongly 2-irreducible if and only if the generator of $I$ is a prime power or the product of two prime powers.

(4) Every singly strongly 2-irreducible ideal is a 2-absorbing primary ideal.

Proof. (1) By Lemma 1.

(2) Suppose that $I$ is singly strongly 2 -irreducible and $p_{1}^{n_{1}} p_{2}^{n_{2}} \cdots p_{k}^{n_{k}} \in I$ in which $p_{i}$ 's are distinct prime elements of $R$ and $n_{i}$ 's are natural numbers. Then $\left[p_{1}^{n_{1}}, p_{2}^{n_{2}}, \ldots, p_{k}^{n_{k}}\right]=p_{1}^{n_{1}} p_{2}^{n_{2}} \cdots p_{k}^{n_{k}} \in I$. Hence by part (1), there are $1 \leq r, s \leq k$ such that $\left[p_{r}^{n_{r}}, p_{s}^{n_{s}}\right] \in I$, i.e., $p_{r}^{n_{r}} p_{s}^{n_{s}} \in I$. 
For the converse, let $[x, y, z] \in I$ for some $x, y, z \in R \backslash\{0\}$. Assume that $x, y$ and $z$ have prime decompositions as below,

$$
\begin{aligned}
& x=p_{1}^{\alpha_{1}} p_{2}^{\alpha_{2}} \cdots p_{k}^{\alpha_{k}} q_{1}^{\beta_{1}} q_{2}^{\beta_{2}} \cdots q_{s}^{\beta_{s}}, \\
& y=p_{1}^{\gamma_{1}} p_{2}^{\gamma_{2}} \cdots p_{k}^{\gamma_{k}} r_{1}^{\delta_{1}} r_{2}^{\delta_{2}} \cdots r_{u}^{\delta_{u}}, \\
& z=p_{1}^{\varepsilon_{1}} p_{2}^{\varepsilon_{2}} \cdots p_{k^{\prime}}^{\varepsilon_{k^{\prime}}} q_{1}^{\lambda_{1}} q_{2}^{\lambda_{2}} \cdots q_{s^{\prime}}^{\lambda_{s^{\prime}}} r_{1}^{\mu_{1}} r_{2}^{\mu_{2}} \cdots r_{u^{\prime}}^{\mu_{u^{\prime}} s_{1}^{\kappa_{1}} s_{2}^{\kappa_{2}} \cdots s_{v}^{\kappa_{v}},}
\end{aligned}
$$

in which $0 \leq k^{\prime} \leq k, 0 \leq s^{\prime} \leq s$ and $0 \leq u^{\prime} \leq u$. Therefore,

$$
\begin{aligned}
{[x, y, z]=} & p_{1}^{\nu_{1}} p_{2}^{\nu_{2}} \cdots p_{k^{\prime}}^{v_{k^{\prime}}} p_{k^{\prime}+1}^{\omega_{k^{\prime}+1}} \cdots p_{k}^{\omega_{k}} q_{1}^{\rho_{1}} q_{2}^{\rho_{2}} \cdots q_{s^{\prime}}^{\rho_{s^{\prime}}} \\
& q_{s^{\prime}+1}^{\beta_{s^{\prime}+1}} \cdots q_{s}^{\beta_{s}} r_{1}^{\sigma_{1}} r_{2}^{\sigma_{2}} \cdots r_{u^{\prime}}^{\sigma_{u^{\prime}}} r_{u^{\prime}+1}^{\delta_{u^{\prime}+1}} \cdots r_{u}^{\delta_{u}} s_{1}^{\kappa_{1}} s_{2}^{\kappa_{2}} \cdots s_{v}^{\kappa_{v}} \in I,
\end{aligned}
$$

where $v_{i}=\max \left\{\alpha_{i}, \gamma_{i}, \varepsilon_{i}\right\}$ for every $1 \leq i \leq k^{\prime} ; \omega_{j}=\max \left\{\alpha_{j}, \gamma_{j}\right\}$ for every $k^{\prime}<$ $j \leq k ; \rho_{i}=\max \left\{\beta_{i}, \lambda_{i}\right\}$ for every $1 \leq i \leq s^{\prime} ; \sigma_{i}=\max \left\{\delta_{i}, \mu_{i}\right\}$ for every $1 \leq i \leq u^{\prime}$. By part (2), we have twenty one cases. For example we investigate the following two cases. The other cases can be verified in a similar way.

Case 1. For some $1 \leq i, j \leq k^{\prime}, p_{i}^{v_{i}} p_{j}^{v_{j}} \in I$. If $v_{i}=\alpha_{i}$ and $v_{j}=\alpha_{j}$, then clearly $x \in I$ and so $[x, y] \in I$. If $v_{i}=\alpha_{i}$ and $v_{j}=\gamma_{j}$, then $p_{i}^{\alpha_{i}} p_{j}^{\gamma_{j}} \mid[x, y]$ and thus $[x, y] \in I$. If $v_{i}=\alpha_{i}$ and $v_{j}=\varepsilon_{j}$, then $p_{i}^{\alpha_{i}} p_{j}^{\varepsilon_{j}} \mid[x, z]$ and thus $[x, z] \in I$.

Case 2. Let $p_{i}^{v_{i}} p_{j}^{\omega_{j}} \in I$; for some $1 \leq i \leq k^{\prime}$ and $k^{\prime}+1 \leq j \leq k$. For $v_{i}=\alpha_{i}$, $\omega_{j}=\alpha_{j}$ we have $x \in I$ and so $[x, y] \in I$. For $\nu_{i}=\varepsilon_{i}, \omega_{j}=\gamma_{j}$ we have $[y, z] \in I$.

Consequently $I$ is singly strongly 2 -irreducible, by part (1).

(3) Suppose that $I=R a$ for some nonzero element $a \in R$. Assume that $I$ is singly strongly 2-irreducible. Let $a=p_{1}^{n_{1}} p_{2}^{n_{2}} \cdots p_{k}^{n_{k}}$ be a prime decomposition for $a$ such that $k>2$. By part (2) we have that $p_{r}^{n_{r}} p_{s}^{n_{s}} \in I$ for some $1 \leq r, s \leq k$. Therefore $I=R\left(p_{r}^{n_{r}} p_{s}^{n_{s}}\right)$.

Conversely, if $a$ is a prime power, then $I$ is strongly irreducible ideal, by [3, Theorem 2.2(3)]. Hence $I$ is singly strongly 2 -irreducible. Let $I=R\left(p^{r} q^{s}\right)$ for some prime elements $p, q$ of $R$. Assume that for some distinct prime elements $q_{1}, q_{2}, \ldots, q_{k}$ of $R$ and natural numbers $m_{1}, m_{2}, \ldots, m_{k}, q_{1}^{m_{1}} q_{2}^{m_{2}} \cdots q_{k}^{m_{k}} \in I=R\left(p^{r} q^{s}\right)$. Then $p^{r} q^{s} \mid q_{1}^{m_{1}} q_{2}^{m_{2}} \cdots q_{k}^{m_{k}}$. Hence there exists $1 \leq i \leq k$ such that $p=q_{i}$ and $r \leq m_{i}$, also there exists $1 \leq j \leq k$ such that $q=q_{j}$ and $s \leq m_{j}$. Then, since $p^{r} q^{s} \in I$, we have $q_{i}^{m_{i}} q_{j}^{m_{j}} \in I$. Now, by part (2), $I$ is singly strongly 2 -irreducible.

(4) Let $I$ be singly strongly 2 -irreducible and $x y z \in I$ for some $x, y, z \in R \backslash\{0\}$. Consider the following prime decompositions,

$$
\begin{aligned}
& x=p_{1}^{\alpha_{1}} p_{2}^{\alpha_{2}} \cdots p_{k}^{\alpha_{k}} q_{1}^{\beta_{1}} q_{2}^{\beta_{2}} \cdots q_{s}^{\beta_{s}}, \\
& y=p_{1}^{\gamma_{1}} p_{2}^{\gamma_{2}} \cdots p_{k}^{\gamma_{k}} r_{1}^{\delta_{1}} r_{2}^{\delta_{2}} \cdots r_{u}^{\delta_{u}}, \\
& z=p_{1}^{\varepsilon_{1}} p_{2}^{\varepsilon_{2}} \cdots p_{k^{\prime}}^{\varepsilon_{k^{\prime}}} q_{1}^{\lambda_{1}} q_{2}^{\lambda_{2}} \cdots q_{s^{\prime}}^{\lambda_{s^{\prime}}} r_{1}^{\mu_{1}} r_{2}^{\mu_{2}} \cdots r_{u^{\prime}}^{\mu_{u^{\prime}}} s_{1}^{\kappa_{1}} s_{2}^{\kappa_{2}} \cdots s_{v}^{\kappa_{v}},
\end{aligned}
$$

in which $0 \leq k^{\prime} \leq k, 0 \leq s^{\prime} \leq s$ and $0 \leq u^{\prime} \leq u$. By these representations we have, 


$$
\begin{gathered}
x y z=p_{1}^{\alpha_{1}+\gamma_{1}+\varepsilon_{1}} p_{2}^{\alpha_{2}+\gamma_{2}+\varepsilon_{2}} \cdots p_{k^{\prime}}^{\alpha_{k^{\prime}}+\gamma_{k^{\prime}}+\varepsilon_{k^{\prime}}} p_{k^{\prime}+1}^{\alpha_{k^{\prime}+1}+\gamma_{k^{\prime}+1}} \\
\cdots p_{k}^{\alpha_{k}+\gamma_{k}} q_{1}^{\beta_{1}+\lambda_{1}} q_{2}^{\beta_{2}+\lambda_{2}} \cdots q_{s^{\prime}}^{\beta_{s^{\prime}}+\lambda_{s^{\prime}}} q_{s^{\prime}+1}^{\beta_{s^{\prime}+1}} \cdots q_{s}^{\beta_{s}} \\
r_{1}^{\delta_{1}+\mu_{1}} r_{2}^{\delta_{2}+\mu_{2}} \cdots r_{u^{\prime}}^{\delta_{u^{\prime}}+\mu_{u^{\prime}}} r_{u^{\prime}+1}^{\delta_{u^{\prime}+1}} \cdots r_{u}^{\delta_{u}} s_{1}^{\kappa_{1}} s_{2}^{\kappa_{2}} \cdots s_{v}^{\kappa_{v}} \in I .
\end{gathered}
$$

Now, apply part (2). We investigate some cases that can be happened, the other cases similarly lead us to the claim that $I$ is 2-absorbing primary. First, assume for some $1 \leq i, j \leq k^{\prime}, p_{i}^{\alpha_{i}+\gamma_{i}+\varepsilon_{i}} p_{j}^{\alpha_{j}+\gamma_{j}+\varepsilon_{j}} \in I$. Choose a natural number $n$ such that $n \geq \max \left\{\frac{\alpha_{i}+\gamma_{i}}{\varepsilon_{i}}, \frac{\alpha_{j}+\gamma_{j}}{\varepsilon_{j}}\right\}$. With this choice we have $(n+1) \varepsilon_{i} \geq \alpha_{i}+\gamma_{i}+\varepsilon_{i}$ and $(n+1) \varepsilon_{j} \geq \alpha_{j}+\gamma_{j}+\varepsilon_{j}$, so $p_{i}^{(n+1) \varepsilon_{i}} p_{j}^{(n+1) \varepsilon_{j}} \in I$. Then $z^{n+1} \in I$, so $z \in$ $\sqrt{I}$. The other one case; assume that for some $1 \leq i \leq k^{\prime}$ and $k^{\prime}+1 \leq j \leq k$, $p_{i}^{\alpha_{i}+\gamma_{i}+\varepsilon_{i}} p_{j}^{\alpha_{j}+\gamma_{j}} \in I$. Choose a natural number $n$ such that $n \geq \max \left\{\frac{\alpha_{i}+\varepsilon_{i}}{\gamma_{i}}, \frac{\alpha_{j}}{\gamma_{j}}\right\}$. With this choice we have $(n+1) \gamma_{i} \geq \alpha_{i}+\gamma_{i}+\varepsilon_{i}$ and $(n+1) \gamma_{j} \geq \alpha_{j}+\gamma_{j}$, thus $p_{i}^{(n+1) \gamma_{i}} p_{j}^{(n+1) \gamma_{j}} \in I$. Then $y^{n+1} \in I$, so $y \in \sqrt{I}$. Assume that $p_{i}^{\alpha_{i}+\gamma_{i}} s_{j}^{\kappa_{j}} \in I$, for some $k^{\prime}+1 \leq i \leq k$ and some $1 \leq j \leq v$. Let $n$ be a natural number where $n \geq \frac{\gamma_{i}}{\alpha_{i}}$, then $(n+1) \alpha_{i} \geq \alpha_{i}+\gamma_{i}$. Hence $p_{i}^{(n+1) \alpha_{i}} s_{j}^{(n+1) \kappa_{j}} \in I$ which shows that $x z \in \sqrt{I}$. Suppose that for some $s^{\prime}+1 \leq i \leq s$ and $u^{\prime}+1 \leq j \leq u, q_{i}^{\beta_{i}} r_{j}^{\delta_{j}} \in I$. Then, clearly $x y \in I$.

Corollary 8. Let $R$ be a $U F D$.

(1) Every principal ideal of $R$ is a singly strongly 2-irreducible ideal if and only if it is a 2-absorbing primary ideal.

(2) Every singly strongly 2-irreducible ideal of $R$ can be generated by a set of elements of the forms $p^{n}$ and $p_{i}^{n_{i}} p_{j}^{n_{j}}$ in which $p, p_{i}, p_{j}$ are some prime elements of $R$ and $n, n_{i}, n_{j}$ are some natural numbers.

(3) Every 2-absorbing ideal of $R$ is a singly strongly 2-irreducible ideal.

Proof. (1) Suppose that $I$ is singly strongly 2-irreducible ideal. By Theorem $10(4), I$ is a 2 -absorbing primary ideal. Conversely, let $I$ be a nonzero 2-absorbing primary ideal. Let $I=R a$, where $0 \neq a \in I$. Assume that $a=p_{1}^{n_{1}} p_{2}^{n_{2}} \cdots p_{k}^{n_{k}}$ be a prime decomposition for $a$. If $k>2$, then since $p_{1}^{n_{1}} p_{2}^{n_{2}} \cdots p_{k}^{n_{k}} \in I$ and $I$ is a 2-absorbing primary ideal, there exist a natural number $n$, and integers $1 \leq i, j \leq k$ such that $p_{i}^{n n_{i}} p_{j}^{n n_{j}} \in I$, say $i=1$ and $j=2$. Therefore $p_{3} \mid p_{1}^{n n_{1}} p_{2}^{n n_{2}}$ which is a contradiction. Therefore $k=1$ or 2 , that is $I=R p_{1}^{n_{1}}$ or $I=R\left(p_{1}^{n_{1}} p_{2}^{n_{2}}\right)$, respectively. Hence by Theorem $10(3), I$ is singly strongly 2 -irreducible.

(2) Let $X$ be a generator set for a singly strongly 2-irreducible ideal of $I$, and let $x$ be a nonzero element of $X$. Assume that $x=p_{1}^{n_{1}} p_{2}^{n_{2}} \cdots p_{k}^{n_{k}}$ be a prime decomposition for $x$ such that $k \geq 2$. By Theorem 10(2), for some $1 \leq i, j \leq k$, we have $p_{i}^{n_{i}} p_{j}^{n_{j}} \in I$, and then $R x \subseteq R p_{i}^{n_{i}} p_{j}^{n_{j}} \subseteq I$. Consequently, $I$ can be generated by a set of elements 
of the forms $p^{n}$ and $p_{i}^{n_{i}} p_{j}^{n_{j}}$.

(3) is a direct consequence of Theorem 10(2).

The following example shows that in part (1) of Corollary 8 the condition that $I$ is principal is necessary. Moreover, the converse of part (2) of this corollary need not be true.

Example 2. Let $F$ be a field and $R=F[x, y, z]$, where $x, y$ and $z$ are independent indeterminates. We know that $R$ is a $U F D$. Suppose that $I=\left\langle x, y^{2}, z^{2}\right\rangle$. Since $\sqrt{\left\langle x, y^{2}, z^{2}\right\rangle}=\langle x, y, z\rangle$ is a maximal ideal of $R, I$ is a primary ideal and so is a 2absorbing primary ideal. Notice that $(x+y+z) y z \in I$, but neither $(x+y+z) y \in I$ nor $(x+y+z) z \in I$ nor $y z \in I$. Consequently, $I$ is not singly strongly 2 -irreducible, by Theorem 10(2).

\section{ACKNOWLEDGMENTS}

The authors are grateful to the referee of this paper for his/her careful reading and comments.

\section{REFERENCES}

[1] D. D. Anderson and D. F. Anderson, "Generalized GCD-domains," Comment. Math. Univ. St. Pauli, vol. 28, pp. 215-221, 1980.

[2] D. F. Anderson and A. Badawi, "On $n$-absorbing ideals of commutative rings," Comm. Algebra, vol. 39, no. 5, pp. 1646-1672, 2011.

[3] A. Azizi, "Strongly irreducible ideals," J. Aust. Math. Soc., vol. 84, no. 2, pp. 145-154, 2008.

[4] A. Badawi, "On 2-absorbing ideals of commutative rings," Bull. Aust. Math. Soc., vol. 75, no. 3, pp. 417-429, 2007.

[5] A. Badawi and A. Y. Darani, "On weakly 2-absorbing ideals of commutative rings," Houston J. Math., vol. 39, no. 2, pp. 441-452, 2013.

[6] A. Badawi, U. Tekir, and E. Yetkin, "On 2-absorbing primary ideals in commutative rings," Bull. Korean Math. Soc., vol. 51, no. 4, pp. 1163-1173, 2014.

[7] A. Y. Darani and H. Mostafanasab, "Co-2-absorbing preradicals and submodules," J. Algebra Appl., vol. 14, no. 7, p. 1550113 (23 pages), 2015.

[8] A. Y. Darani and H. Mostafanasab, "On 2-absorbing preradicals," J. Algebra Appl., vol. 14, no. 2, p. 1550017 (22 pages), 2015.

[9] A. Y. Darani and E. R. Puczyłowski, "On 2-absorbing commutative semigroups and their applications to rings," Semigroup Forum, vol. 86, no. 1, pp. 83-91, 2013.

[10] A. Y. Darani and F. Soheilnia, "2-absorbing and weakly 2-absorbing submoduels," Thai J. Math., vol. 9, no. 3, pp. 577-584, 2011.

[11] A. Y. Darani and F. Soheilnia, "On $n$-absorbing submodules," Math. Commun., vol. 17, no. 2, pp. 547-557, 2012.

[12] L. Fuchs and L. Salce, Modules over non-noetherian domains, ser. Mathematical Surveys and Monographs. United States of America: American Mathematical Society, 2001, vol. 84.

[13] W. J. Heinzer, L. J. Ratliff, and D. E. Rush, "Strongly irreducible ideals of a commutative ring," J. Pure Appl. Algebra, vol. 166, no. 3, pp. 267-275, 2002, doi: 10.1016/S0022-4049(01)00043-3.

[14] T. W. Hungerford, Algebra, ser. Graduate Texts in Mathematics. New York, Heidelberg, Berlin: Springer-Verlag, 1980, vol. 73. 
[15] C. U. Jensen, "Arithmetical rings," Acta Math. Acad. Sci. Hung., vol. 17, pp. 115-123, 1966, doi: 10.1007/BF02020446.

[16] Y. C. Jeon, N. K. Kim, and Y. Lee, "On fully idempotent rings," Bull. Korean Math. Soc., vol. 47, no. 4, pp. 715-726, 2010, doi: 10.4134/BKMS.2010.47.4.715.

[17] T. Y. Lam, Lectures on modules and rings, ser. Graduate Texts in Mathematics. New York: Springer-Verlag, 1999, vol. 189.

[18] D. Lorenzini, An invitation to arithmetic geometry, ser. Graduate Texts in Mathematics. United States of America: American Mathematical Society, 1995, vol. 9.

[19] H. Mostafanasab, E. Yetkin, U. Tekir, and A. Y. Darani, "On 2-absorbing primary submodules of modules over commutative rings," An. Stiint. Univ. "Ovidius" Constanta, Ser. Mat., in press.

[20] R. Sharp, Steps in commutative algebra, 2nd ed., ser. Graduate Texts in Mathematics. London: London Mathematical Society Student Texts, Cambridge University Press, 2000, vol. 51.

\section{Authors' addresses}

H. Mostafanasab

University of Mohaghegh Ardabili, Department of Mathematics and Applications, P. O. Box 179, Ardabil, Iran

E-mail address: h.mostafanasab@uma.ac.ir; h.mostafanasab@gmail.com

\section{A. Yousefian Darani}

University of Mohaghegh Ardabili, Department of Mathematics and Applications, P. O. Box 179, Ardabil, Iran

E-mail address: yousefian@uma.ac.ir, youseffian@gmail.com 